\title{
Can YAG screen accept LEReC bunch train?
}

\author{
S. Seletskiy, P. Thieberger, T. Miller
}

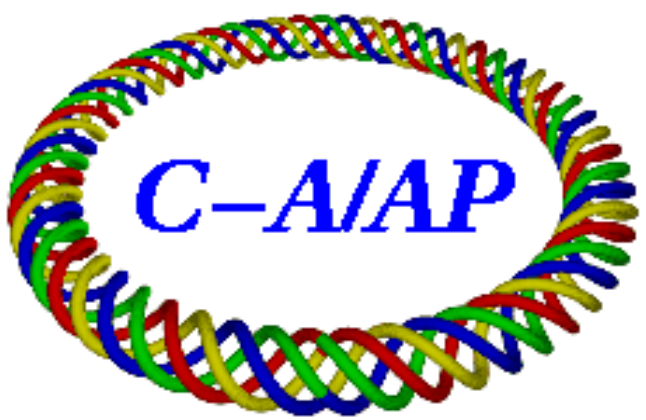

Collider-Accelerator Department Brookhaven National Laboratory

Upton, NY 11973

\section{U.S. Department of Energy Office of Science, Office of Nuclear Physics}

Notice: This document has been authorized by employees of Brookhaven Science Associates, LLC under Contract No. DE-SC0012704 with the U.S. Department of Energy. The United States Government retains a nonexclusive, paid-up, irrevocable, world-wide license to publish or reproduce the published form of this document, or allow others to do so, for United States Government purposes. 


\title{
Can YAG screen accept LEReC bunch train?
}

\author{
May 18, 2016 \\ S. Seletskiy, P. Thieberger, T. Miller
}

\section{Introduction}

LEReC RF diagnostic beamline is supposed to accept 250 us long pulse trains of $1.6 \mathrm{MeV}-$ 2.6 MeV (kinetic energy) electrons. This beamline is equipped with YAG profile monitor.

Since we are interested in observing only the last macro bunch in the train, one of the possibilities is to install a fast kicker and a dedicated dump upstream of the YAG screen (and related diagnostics equipment). This approach is expensive and challenging from engineering point of view.

Another possibility is to send the whole pulse train to the YAG screen and to use a fast gated camera (such as Imperex B0610 with trigger jitter under 60ns [1]) to observe the image from the last pulse only.

In this paper we study the feasibility of the last approach.

\section{RF diagnostic beamline and pulse train}

The beamline dedicated to RF diagnostics is schematically shown in Fig. 1.

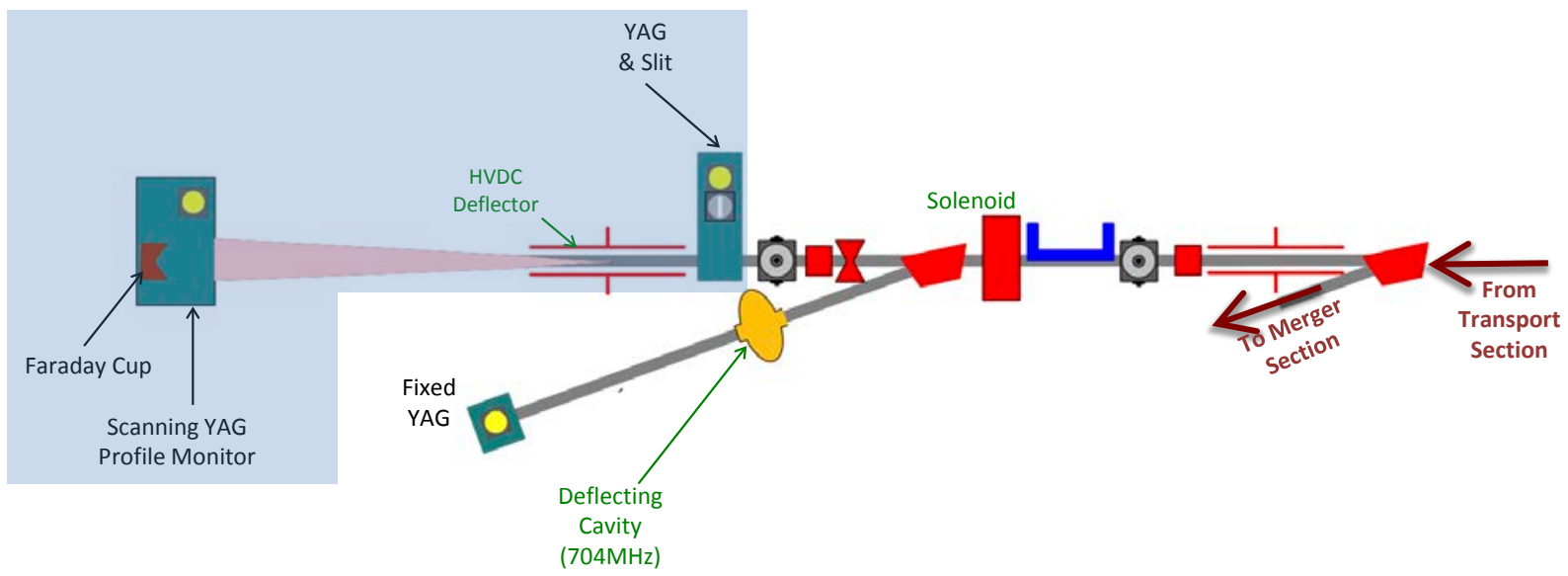

Figure 1: RF diagnostic beamline. Shaded area shows a back-up absolute energy measurements not present in the baseline design.

The beamline consists of a bending magnet creating dispersion at the location of the YAG screen and a deflecting cavity "crabbing" electron bunches in time domain. Thus, the beam image on the YAG screen represents the longitudinal phase space of the beam. 
There is also a potential second branch of the RF diagnostic beamline (shaded in Fig. 1). That branch serves as a back-up plan for an absolute beam energy measurement. It is not present in a baseline design and will be considered only briefly in our analysis.

The temporal structure of the pulse train sent to the diagnostic beamline is as follows. There are $N=30$ (100 ps long) electron bunches, with charge up to $Q=200 \mathrm{pC}$ (for highest kinetic energy beam), spaced by 1.4 ns and forming a single macro bunch. The macro bunches are separated by $\Delta t=110 \mathrm{~ns}$ and form the pulse train of any chosen length. The schematic of ebeam temporal structure is shown in Fig. 2. The overall required length of the pulse train is determined by stabilization time of the RF system. It was determined [2] that pulse train of length $t=250$ us is sufficiently long to study the beam-loading effects in LEReC RF cavities.

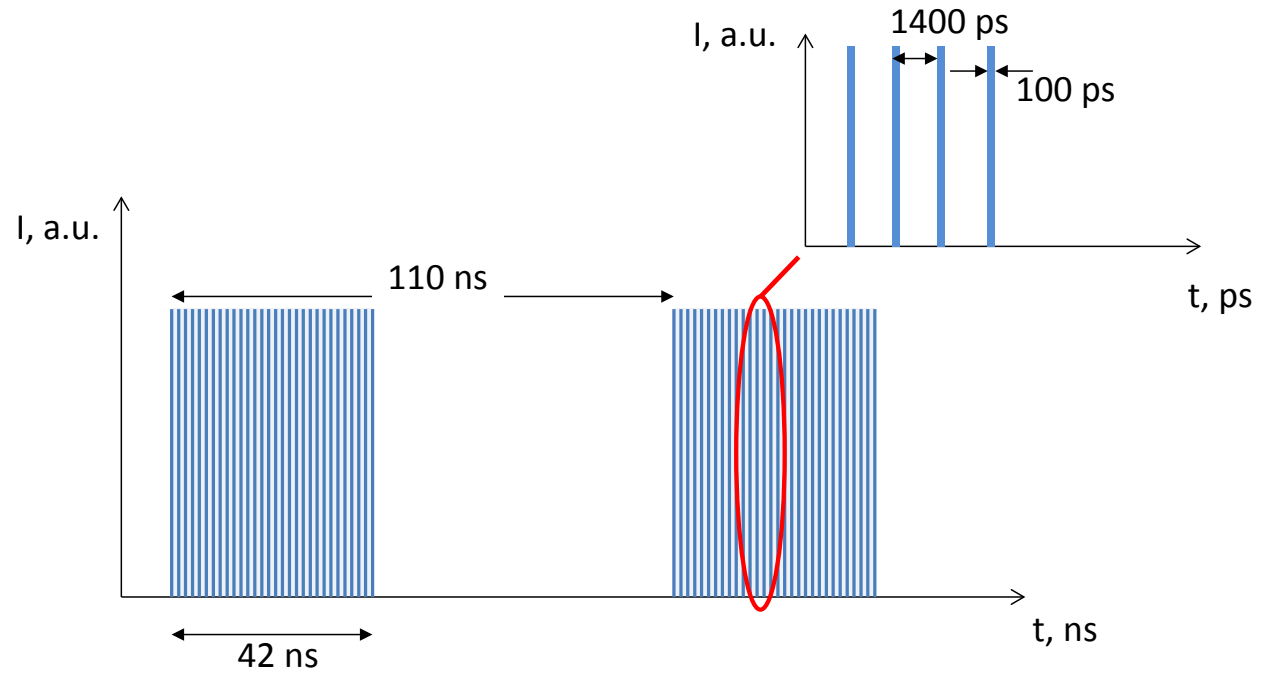

Figure 2: Temporal structure of electron beam.

We will consider two connected questions of YAG screen performance for the described pulse train. First, we will study the instantaneous temperature jump in YAG crystal due to deposition of a single pulse train. Second, we will find the steady-state temperature of YAG screen for various e-beam repetition rates.

\section{What we know about YAG screen performance}

The best, known to us, test of YAG screen performance under temperature stress is the RHIC electron lens operation. In this test $5 \mathrm{keV}, 97 \mathrm{~mA} \mathrm{DC}$ beam "chunks" as long as $1.2 \mathrm{~ms}$ were deposited on the YAG screen.

The crystal response still was linear for such parameters and the Gaussian transverse distribution with $\sigma=0.133 \mathrm{~cm}$ was still observed on the YAG screen.

We calculated that due to deposition of each shot YAG temperature had to jump by $194 \mathrm{~K}$. Numerous shots with 3-5 s repetition rate were sent to YAG crystal without damaging it. 
The test [3] of the dependence of YAG crystal performance on temperature (as shown in Fig. 3) corroborates that it is safe to heat the YAG screen up to $200 \mathrm{C}$.

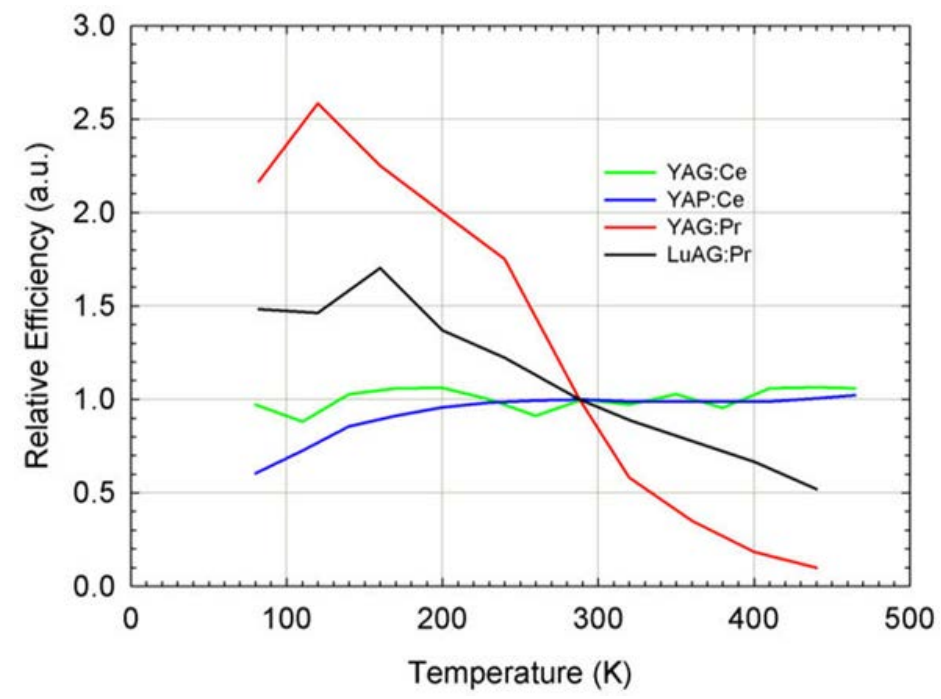

Figure 3: YAG performance depending on crystal temperature.

\section{Instantaneous temperature rise for YAG screen in LEReC RF diagnostic beamline}

YAG stopping power per electron with $2.1 \mathrm{MeV}$ energy is $p=1.46 \mathrm{MeV} \mathrm{cm}^{2} / \mathrm{g}$ [4] (in our energy range it changes only slightly). Therefore, the energy loss per electron in YAG screen of width $w=100$ um is:

$$
\Delta E=p \cdot w \cdot \rho=0.66 \mathrm{MeV}
$$

where $\rho=4.55 \mathrm{~g} / \mathrm{cm}^{3}$ is YAG density.

The total number of electrons interacting with YAG screen is:

$$
N_{e}=N \frac{Q}{e} \frac{t}{\Delta t}=5.54 \cdot 10^{13}
$$

where $e$ is charge of electron.

Thus, from (1) and (2), the total energy deposited on YAG crystal by the pulse train is:

$$
E_{\text {tot }}=N_{e} \Delta E=0.59 \mathrm{~J}
$$

The typical beam profile on YAG screen is shown in Fig. 3. For the sake of energy density calculation we can substitute real beam profile with a homogenous ellipse with $0.11 \mathrm{~cm}$ and $1.79 \mathrm{~cm}$ semi-axes (see Fig. 4 for explanations). 
Since, the specific heat capacity of YAG is $c_{s}=590 \mathrm{~J} / \mathrm{kg} / \mathrm{K}$ [3], the heat capacity of YAG heated by electron beam is $\mathrm{C}=0.017 \mathrm{~J} / \mathrm{K}$.

Finally, an instantaneous increase in YAG temperature due to deposition of a single pulse train is:

$$
\Delta T=\frac{E_{\text {tot }}}{C}=35.5 \mathrm{~K}
$$

Apparently, such small (as compared to electron lens experience) temperature jump will not degrade YAG performance.

Similar calculations for YAG screen in energy measurement part of RF diagnostic beamline give $\Delta T=66 \mathrm{~K}$.
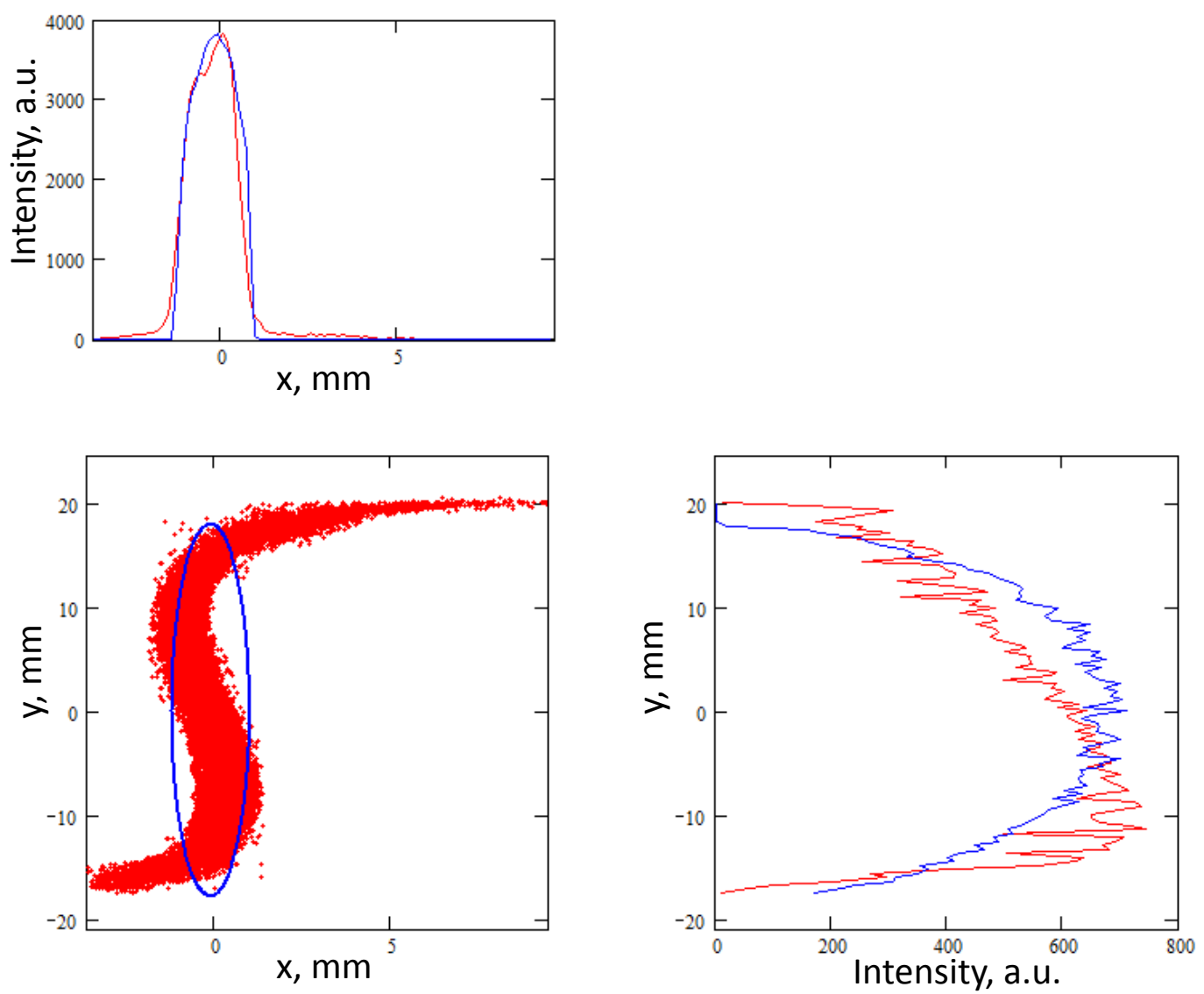

Figure 4: Typical beam profile on YAG screen (red) and equivalent ellipse (blue) homogeneously filled with the same number of particles. For the ellipse, only the outline is shown on the plot.

\section{Steady state temperature of YAG screen in LEReC RF diagnostic beamline}

Assuming that the main mechanism of YAG cooling is radiation we find from black-body radiation formula that the steady state temperature is given by: 


$$
T=\left(\frac{E_{t o t} f}{A \sigma_{S B}}\right)^{\frac{1}{4}}
$$

Here $A$ is the area covered by the beam image; $f$ is the repetition rate of the beam trains, and Stefan-Boltzmann constant $\sigma_{S B}=5.67 \cdot 10^{-8} \mathrm{~W} / \mathrm{m}^{2} / \mathrm{K}^{4}$. We assume $100 \%$ emissivity for the YAG crystal in Eq. 5.

The result of application of formula (5) to our case is shown in Fig. 5. It is obvious that the safe repetition rate can be at least one shot per 5 seconds.

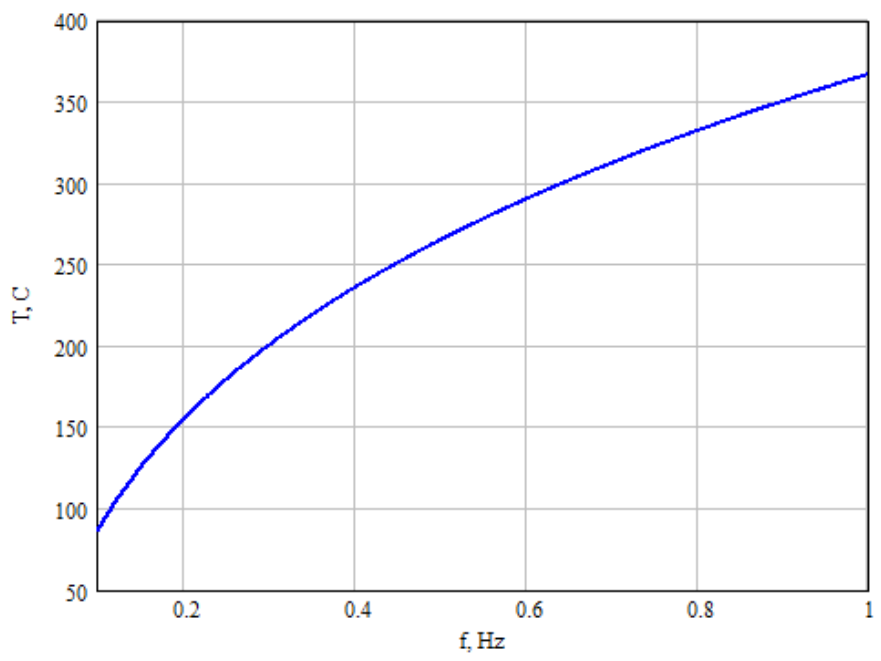

Figure 5: Steady state temperature of YAG crystal depending on e-beam repetition rate.

More precise simulations of YAG cooling take into account crystal emissivity $(\epsilon=0.8)$. To increase the YAG emissivity we plan to coat the back side of the crystal with a layer of carbon on top of the $100 \mathrm{~nm}$ of aluminum. In our simulations we assume that over time period $d t$ the crystal is cooled by temperature:

$$
d T=\frac{\sigma_{S B} \epsilon\left(T^{4}-T_{\text {room }}{ }^{4}\right) d t}{c_{s} \rho w}
$$

where $T_{\text {room }}=300 \mathrm{~K}$ is room temperature.

Results of simulations for pulse train repetition rate of $0.2 \mathrm{~Hz}$ are shown in Fig. 6.

The average steady state temperature of YAG screen for pulse trains arriving with $5 \mathrm{~s}$ period is about $170 \mathrm{C}$ and the maximum observed temperature is $186 \mathrm{C}$.

Please notice that by not exceeding $194 \mathrm{C}$ threshold for steady state temperature we create a rather large safety margin since average steady state temperature in electron lens test had to be about $400 \mathrm{C}$ (calculations for e-lens parameters were performed using Eqs. (1)-(6)). 


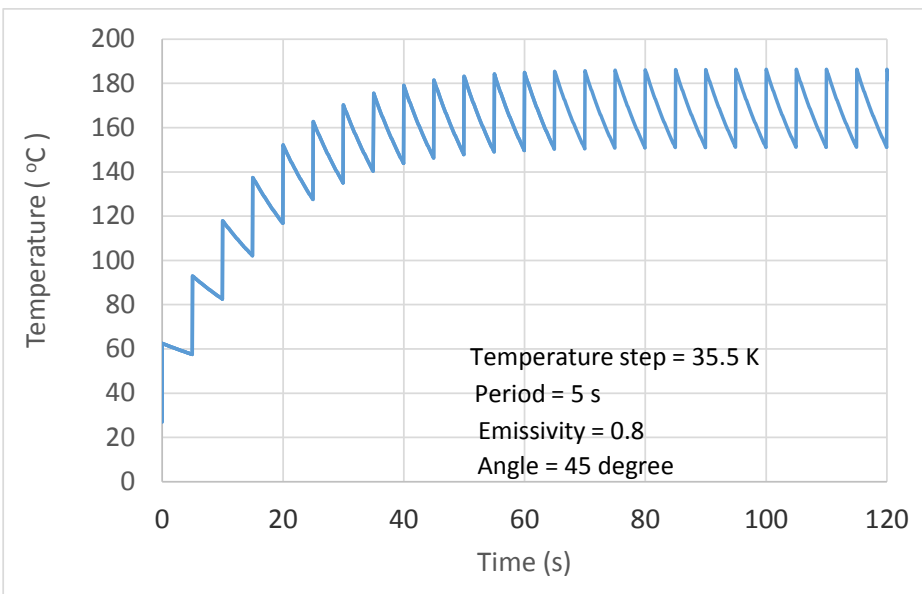

Figure 6: Simulations of steady state temperature.

Similar simulations for YAG screen in energy measurement part of diagnostic beamline gives maximum temperature of $190 \mathrm{C}$ in steady state for beam repetition rate of $0.1 \mathrm{~Hz}$.

Finally, apparently, one can measure longer than 250 us pulse trains by increasing the period between the trains. Figure 7 shows the period between the pulse trains of various length required to guarantee that YAG steady state temperature does not exceed $186 \mathrm{C}$ (maximum temperature for nominal 250 us long pulse train arriving every $5 \mathrm{~s}$ ).

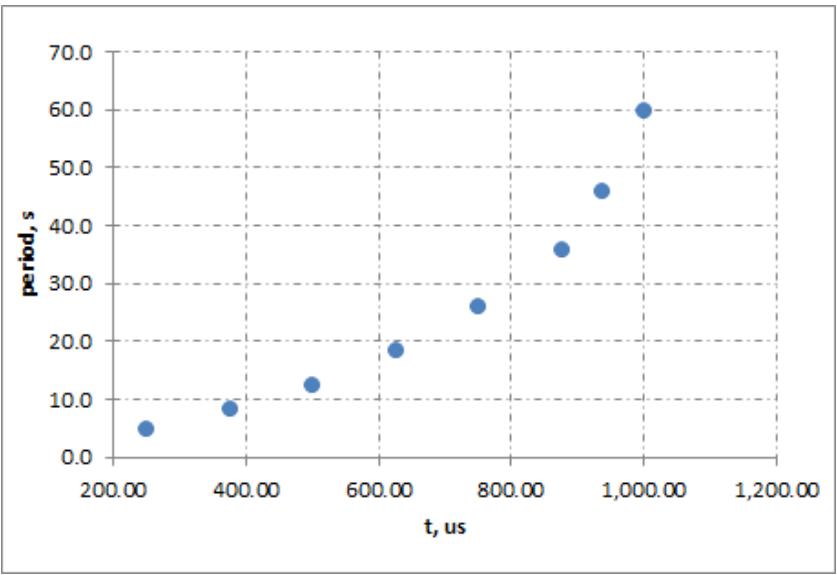

Figure 7: Period between pulse trains depending on train length. For each case YAG steady state temperature does not exceed $186 \mathrm{C}$.

\section{Conclusion}

We considered performance of YAG screen in RF diagnostic beamline for the case of high power (250 us long) pulse trains directed to this screen. We determined that it is absolutely safe to operate such diagnostic with pulse trains repetition rate of $0.2 \mathrm{~Hz}$. 
If eventually we need to build the "electrostatic spectrometer energy measurement" part of the diagnostic beamline then the YAG monitor there can be safely operated with $0.1 \mathrm{~Hz}$ repetition rate.

\section{References}

[1] T. Naito, T.M. Mitsuhashi, TUPD08, Proceedings of IBIC2014, Monterey, CA, USA

[2] Private conversations with K. Smith, K. Mernick, A. Zaltsman.

[3] Reported by Crytur in email correspondence

[4] http://physics.nist.gov/PhysRefData/Star/Text/ESTAR-u.html 